\title{
Evidence for a Shift From Place Navigation to Directional Responding in One Variant of the Morris Water Task
}

\author{
Derek A. Hamilton, Katherine G. Akers, \\ Travis E. Johnson, James P. Rice, and \\ Felicha T. Candelaria \\ The University of New Mexico
}

\author{
Edward S. Redhead \\ University of Southampton
}

\begin{abstract}
Previous work from our laboratory has demonstrated that rats display a preference for directional responding over place navigation in a wide range of procedural variants of the Morris water task (Hamilton, Akers, Weisend, \& Sutherland, 2007; Hamilton et al., 2008). A preference for place navigation has only been observed when the pool is reduced as a cue by filling it with water. Studies using dry land mazes have suggested that rats place navigate early in training and later switch to other forms of responding (e.g., motor). The present study evaluated whether rats switch from place navigation to directional responding in the "full-pool" variant of the water task. Rats were given 12, 24, or 36 hidden platform training trials. Probe trials with the pool repositioned in the room revealed a preference for place navigation in rats given 12 trials, an equal division of response preferences in rats given 24 trials, and a preference for directional responding in rats given 36 trials. These results indicate that the early preference for place navigation in the full-pool water task is transient and yields to a preference for directional responding with continued training.
\end{abstract}

Keywords: spatial learning, cognitive mapping, hippocampus, water maze

Supplemental materials: http://dx.doi.org/10.1037/a0013260.supp

The basic behavioral and psychological processes involved in navigation have been extensively studied and debated for many years. Despite considerable disagreement regarding the precise nature of the processes involved in spatial navigation, most researchers have agreed that navigation from one place to another can be achieved by a variety of responses depending on the available interoceptive (e.g., proprioceptive and vestibular) and exteroceptive (e.g., visual cues) sources of control (O'Keefe \& Nadel, 1978; Restle, 1957; Sutherland \& Hamilton, 2004; Tolman, 1948; Watson, 1907). If several distinct sources of control and associated responses can support navigation to a goal location, they could, in principle, operate simultaneously and in parallel. Although the types of navigation that could be effective in a particular situation are generally not mutually exclusive, the possibility that one form of navigation predominates other equally effective forms of navigation has been confirmed by a number of studies (Chamizo, Aznar-Casanova, \& Artigas, 2003; Chamizo, Sterio, \& Mackintosh, 1985; Hamilton, Rosenfelt, \& Whishaw, 2004; Redhead, Roberts, Good, \& Pearce, 1997; Roberts \& Pearce, 1999). Of particular importance for the present study are situations in which the predominant form of navigation changes as a function of the

Derek A. Hamilton, Katherine G. Akers, Travis E. Johnson, James P. Rice, and Felicha T. Candelaria, Department of Psychology, The University of New Mexico; Edward S. Redhead, School of Psychology, University of Southampton.

We thank Jeani Sarosy for assistance with preparation of the manuscript.

Correspondence concerning this article should be addressed to Derek A. Hamilton, MSC03 2220, Department of Psychology, University of New Mexico, Albuquerque, NM, 87131. E-mail: dahamilt@unm.edu amount of training. For example, Packard and McGaugh (1996) found that rats navigated to the reinforced arm of a T-maze on the basis of its spatial location relative to extramaze visual cues early during training, but later switched to performing a simple motor response even though both responses would result in reinforcement with equal success at any point in training (see Chang and Gold, 2003, for a similar result). This result is consistent with earlier data reported by Tolman, Ritchie, and Kalish (1946) who utilized two separate release arms in a T-maze and systematically manipulated the relationship between the release arm and the reinforced arm. Rats that were reinforced for navigating to the spatial location of reinforcement (requiring different motor responses from trial to trial) quickly learned the appropriate response, whereas rats that were reinforced for making a simple motor response (requiring navigation to different locations) learned much more slowly. Collectively, these observations have been taken as evidence that navigation based on spatial cognitive maps is established more rapidly than simple motor responses, and that the former yields to the latter with continued training.

The conclusions reached by Tolman et al. (1946) and Packard and McGaugh (1996) have served as a point of departure for many subsequent studies, however, whether the results reflect a preference for true place navigation early in training has been questioned. Blodgett, McCutchan, and Mathews (1949) noted that the apparent place responding reported by Tolman et al. could also be achieved by navigating in the direction of reinforcement within the room reference frame. By rotating and/or translating the maze on each training trial Blodgett et al. were able to make consistent motor responding, place responding, and directional responding mutually exclusive, with only one response form resulting in 
reinforcement. Simple motor responses and directional responses were learned rather easily whereas place responses were acquired with great difficulty. Later, Skinner et al. (2003) replicated the findings of Blodgett et al. in the T-maze and in an open field and concluded that the results of Packard and McGaugh likely reflect an early preference for directional responding rather than a preference for true place navigation.

Following the initial work of Weisend et al. (1995), Hamilton and colleagues (Akers, Candelaria, \& Hamilton, 2007; Hamilton et al., 2008; Hamilton, Akers, Weisend, \& Sutherland, 2007) trained rats to navigate to an escape platform in a fixed location in the Morris water task (Morris, 1981) after which the pool was repositioned in the room such that directional responding and true place navigation would result in navigation to distinct locations in the pool. Directional responding predominated true place navigation in all situations in which the pool wall was a prominent feature of the environment, as is the case in the water task as it is typically used. More important, simple motor responses or route learning are not effective in the standard water task because multiple-release points that vary in distance and direction from the platform are utilized. Based on the general implications of Packard and McGaugh's (1996) observations concerning a shift from place navigation to other forms of navigation, Hamilton et al. $(2007,2008)$ evaluated whether a preference for true place navigation was subsequently replaced by a preference for directional responding in the water task. The basic preference for directional responding was observed after minimal active swim training ( 8 trials), extensive training (240 trials), and after a single 30 -s passive platform placement trial, thus, there was little to suggest that rats switch from place navigation to directional responding as a function of the amount of training. A preference for place navigation over directional responding was observed when the pool wall was substantially reduced as a source of control by filling it nearly to the top with opaque water (Hamilton et al., 2008), however, this preference was only evaluated after 12 to 24 training trials. Given that asymptotic levels of performance were reached in 12 to 20 trials this observation can reasonably be considered to reflect a preference for place navigation early during training. Whether this preference yields to a preference for directional responding later in training is addressed by the present study. Rats were given varying amounts of training with the pool wall virtually eliminated after which the pool was repositioned to evaluate the relative preference for place navigation or directional responding. Given that the T-maze studies of Packard and McGaugh and Chang and Gold (2003) did not rule out an early preference for directional responding, the present study could provide the first unambiguous demonstration of a switch from true place navigation to another form of navigation with continued training. If such a switch is not observed then the generality of the results provided by Packard and McGaugh can be questioned, and the utility of the procedures utilized by Hamilton et al. (2008) for establishing a robust and persistent preference for true place navigation in the water task will be more firmly substantiated.

\section{Method}

\section{Subjects}

Subjects were 32 naïve male Long-Evans rats (Charles River Laboratories, Wilmington, MA) that were approximately 90 days old at the beginning of the experiment. All rats were pair housed in plastic cages on a 12-hr light:dark cycle with food and water available ad libitum. Behavioral testing was performed during the light phase. Procedures for the studies reported here were approved by the Institutional Animal Care and Use Committee at the University of New Mexico.

\section{Apparatus}

The pool and room were the same as those used by Hamilton et al. (2008) in their Experiment 6. The testing room contained a number of distal visual cues (e.g., posters, a chalk board, the experimenter) and the room walls formed a complex geometry (see Figure 1). The circular pool (1.5 m diameter, $46 \mathrm{~cm}$ high) was placed on a wooden frame $(48 \mathrm{~cm}$ tall) that rested on appliance rollers so that the pool could be moved. The pool was filled to a depth of $42 \mathrm{~cm}$ with cool water $\left(22^{\circ} \mathrm{C}\right)$ that was made opaque by a small amount of nontoxic, white tempura paint, leaving only 4 $\mathrm{cm}$ of the pool wall visible. To prevent escape from the pool, four sections of clear Plexiglas $(3 \mathrm{~mm}$ thick, $16 \mathrm{~cm}$ high, $118 \mathrm{~cm}$ in length) were mounted to the inner surface of the pool wall such that the Plexiglas closely conformed to the circular geometry of the pool. The joints where the Plexiglas sections met were identical and equally spaced around the pool so as not to provide any information about the precise location of the platform. The Plexiglas sections extended $17 \mathrm{~cm}$ above the surface of the water and were, therefore, visible however; they were transparent and did not obscure the distal visual environment. The top of the platform was $16 \mathrm{~cm} \times 16 \mathrm{~cm}$ and was approximately $1 \mathrm{~cm}$ below the water's surface. Digital video of each trial was captured via an overhead camera and transferred to a Linux workstation for tracking and analysis.

\section{Design and Procedure}

Rats were randomly assigned in equal numbers $(n=8)$ to one of four groups: No Shift, Shift-12, Shift-24, or Shift-36. Rats in the No Shift and Shift-12 groups were given 12 hidden platform training trials (three blocks of four trials), Shift-24 rats were given 24 trials (six blocks over 2 days), and Shift-36 rats were given 36 trials (nine blocks over 3 days). The selection of 12 trials as the minimum amount of training was based on prior work showing that rats do not begin to take direct trajectories to the platform until 10 to 12 training trials have been given (Hamilton et al., 2008). Two pool positions separated by $75 \mathrm{~cm}$ were used were used during training. Half the rats from each group were trained with the pool at Position 1 (see Figure 1) and the other half were trained with the pool at Position 2. The pool remained in the same position throughout training and the platform was always in the same location in the room (Location B, see Figure 1) regardless of the pool position. On each trial rats were released at one of four release points (NW, SW, SE, NE) around the perimeter of the pool. The release points were selected pseudorandomly without replacement so that each release point was used once during each block of four trials. Latency to navigate to the platform served as the dependent measure. Rats were removed from the escape platform after 5 to $10 \mathrm{~s}$ and returned to a holding cage for an intertrial interval of approximately 3 to $5 \mathrm{~min}$. In cases in which a rat did not navigate to the platform within $60 \mathrm{~s}$ it was retrieved by the experimenter and 

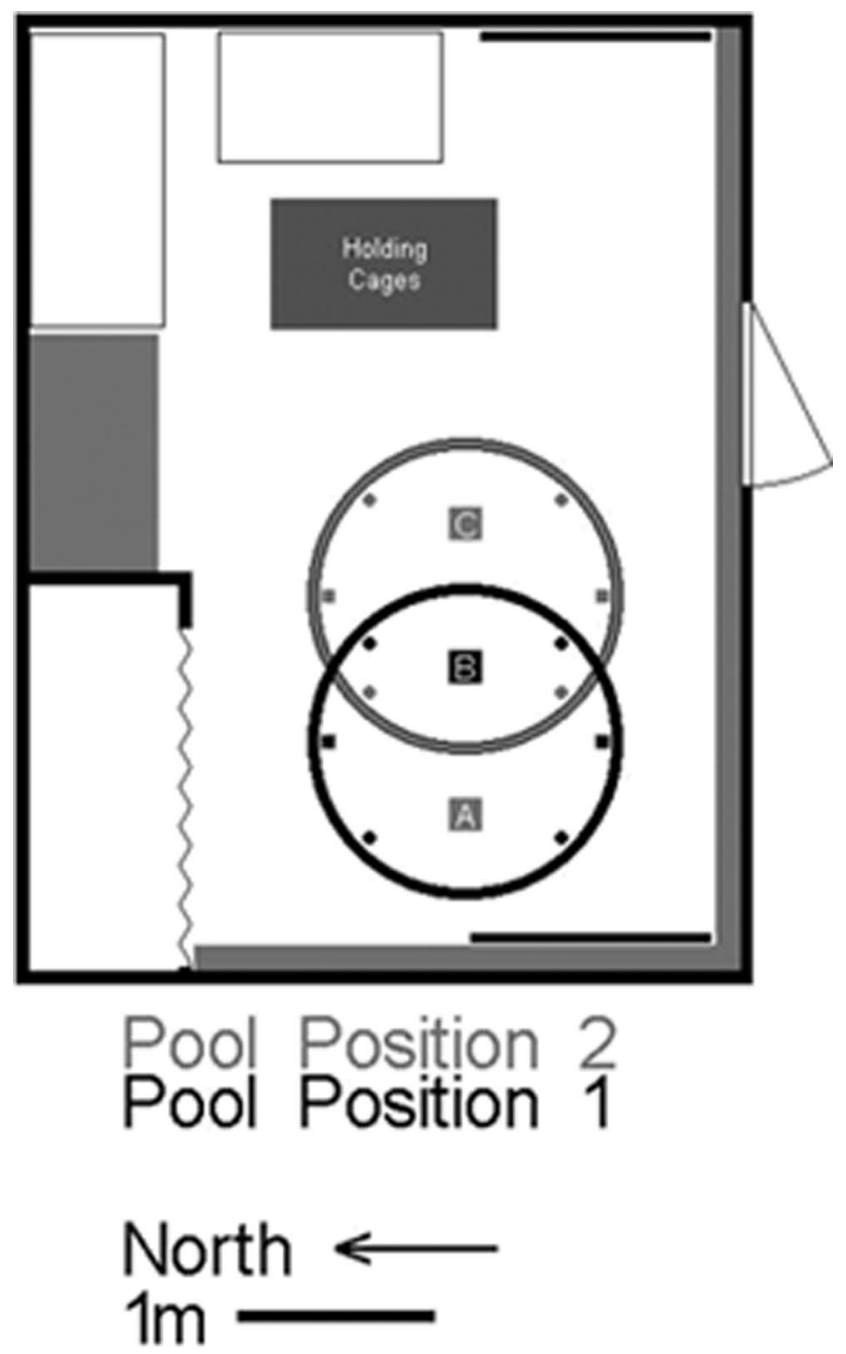

Figure 1. Layout of the testing room showing the room geometry and location of prominent visual cues (gray or black rectangles). The pool was located in one of two positions that were separated by $75 \mathrm{~cm}$ (the pool radius). The escape platform was always placed at Location $\mathrm{B}$, which represents the same absolute spatial location within the room reference frame for both pool positions. Locations $\mathrm{A}$ and $\mathrm{C}$ represent comparison locations that are in the opposite quadrant from the platform Location (B) for pool Positions 1 and 2, respectively. For the shift groups these locations correspond to the relative location of the platform within the pool. The dark circles inside the pool mark the four release points used during hidden platform training and the squares represent the two release points used for the no platform probe trial.

placed directly on the platform for 5 to $10 \mathrm{~s}$ before being returned to its cage. On each day rats were given three blocks of 4 trials (12 trials total), thus, the number of days over which the rats were trained differed among the shift groups (see above). We note that only one level of training (12 trials) was used for No Shift animals. This decision was based on several factors: (a) Pilot work revealed that 12 trials was sufficient to achieve asymptotic levels of performance (also see the training results below), (b) 12 training trials is sufficient to achieve good performance on probe trials when the pool remains in the same location used during training, (c) the basic preference for the absolute location on probe trials when the pool remains in the same location as used during training does not change as function of training trials (Hamilton et al., 2008), (d) 12 trials represents the least amount of training used for any of the shift groups, and (e) the performance of No Shift animals after 12 trials should provide the most conservative estimate of location preference for use in statistical comparisons with the performance of all shift rats.

For the critical test trial the platform was removed from the pool and rats were allowed to swim for $30 \mathrm{~s}$. All rats were released from one of two points ( $\mathrm{N}$ or $\mathrm{S}$ ) selected pseudorandomly with the constraint that each release point was used twice for each combination of pool position and group. The pool was positioned at the same location used during training for rats in the No Shift group. For rats in the shift groups (Shift-12, Shift-24, and Shift-36) the pool was relocated to the position that was not used during training (i.e., if the pool was at Position 1 during training it was moved to Position 2, and vice versa). Four dependent measures were taken for each of two critical locations that were the same size as the platform surface. One critical location was the absolute location of the platform in the room and the other was an equal distance from the pool wall in the diametrically opposite quadrant. When the pool is repositioned, the opposite location corresponds to the same relative location of the platform in the pool during training and is the location to which a directional response would be expected. If the pool is not repositioned, the opposite location serves as a comparison location that has the same spatial relationship to the absolute location as does the relative location for conditions in which the pool is repositioned. For example, if the pool was in Position 1 and the platform was in Location B (see Figure 1) during training, and the pool remained in Position 1 for the probe trial, the opposite location would correspond to Location A. If the pool was repositioned (to Position 2) for the probe then Location $\mathrm{B}$ corresponds to the absolute location and Location $\mathrm{C}$ corresponds to the relative/opposite location. The number of times each critical location was crossed and the average distance from each location during the probe trial were measured. The latter measure was adapted from the goal proximity measure described by Gallagher, Burwell, and Burchinal (1993). The latency to enter and the amount of time spent in a circular region $(66 \mathrm{~cm}$ in diameter) centered on each of the critical locations were also measured (Akers et al., 2007; Hamilton et al., 2007, 2008).

\section{Results}

All statistical tests reported below were significant at $p<.05$ unless otherwise noted.

\section{Hidden Platform Training}

Latencies for each rat were averaged for each block of 4 training trials. Rats in all four groups learned to take direct trajectories to the hidden platform in about 10 to 12 trials. Because the number of training trials varied for each group, separate repeated measures analyses of variances (ANOVAs) were conducted for each group with trial block as a single factor. There were significant main effects of trial block for all four groups, all $p s<.001$, that were attributable to decreases in escape latency across trial blocks. Mean escape latencies during the first trial block ranged from $23.28 \mathrm{~s}$ (Shift-24) to $30.97 \mathrm{~s}$ (No Shift), however, the group effect 
for Block 1 was not significant, $F(3,28)=1, p=.41$. Mean latencies during the final trial block were comparable for the four groups, ranging from $4.35 \mathrm{~s}$ (Shift-36) to $6.25 \mathrm{~s}$ (Shift-12), and did not significantly differ, $F(3,28)=1.31, p=.29$. Hamilton et al. (2008) found that learning in the full-pool task required three blocks of training to reach asymptotic levels of performance compared to the two blocks of training needed in the standard water task. Consistent with this observation, rats in the present experiment took, on average, $27.04 \mathrm{~s}$ to locate the platform during Block $1,15.23 \mathrm{~s}$ to locate the platform in Block 2, and $6.73 \mathrm{~s}$ to locate the platform in Block 3. A repeated-measures ANOVA on these data revealed a significant effect of trial block, $F(2,62)=44.36$. Latencies for Block 2 were significantly lower than latencies for Block 1, and latencies for Block 3 were significantly lower than latencies for Block 2, both $p$ s $<.001$. Latencies for Block 3 did not significantly differ from those of any subsequent blocks (for the Shift-24 and Shift-36 groups), all $p$ s $>.09$.

\section{No-Platform Probe Trial}

Data from the no-platform probe trial are shown in Figure 2. Rats in the No Shift group displayed a clear preference for the absolute location. For the shift groups, the data suggest an initial, weak preference for the absolute location that systematically shifted to a preference for the relative location with additional training. Specifically, a weak preference for the absolute location was observed the Shift-12 group, no systematic preference for either location was observed for the Shift-24 group, and a clear preference for the relative location was observed for the Shift-36 group. The following sets of analyses were undertaken to evaluate (a) the preference for the two critical regions within each group and (b) whether these preferences changed as a function of the amount of training.

All 8 rats in the No Shift group showed a preference for the absolute location over the opposite location as was evident in all of the probe trial swim paths (a representative swim path is shown in the Supplementary Figure). Statistical comparisons of the absolute and opposite location measures (see Figure 2) confirmed these impressions; No Shift rats entered the absolute region faster than the opposite location, $F(1,7)=9.15$, spent more time in the absolute region, $F(1,7)=18.22$, crossed the absolute location more frequently than the opposite location, $F(1,7)=16.61$, and navigated closer to the absolute location, $F(1,7)=9.07$. These data confirm that 12 training trials are sufficient to yield a clear preference for the trained platform location when the pool is not a prominent feature of the environment.

As a group, Shift-12 rats generally showed a preference for the absolute location over the relative location (see Figure 2). A demonstration of preference at the group level is hindered by the fact that 1 rat showed a clear preference for the relative location, whereas the other 7 rats showed a preference for the absolute location that was much weaker in magnitude than the location preferences we have previously observed when the pool wall is a prominent cue (see Hamilton et al., 2007; 2008). The swim path for the single rat that preferred the relative location and a representative swim path for a rat that preferred the absolute location are shown in the Supplementary Figure. Although the numerical
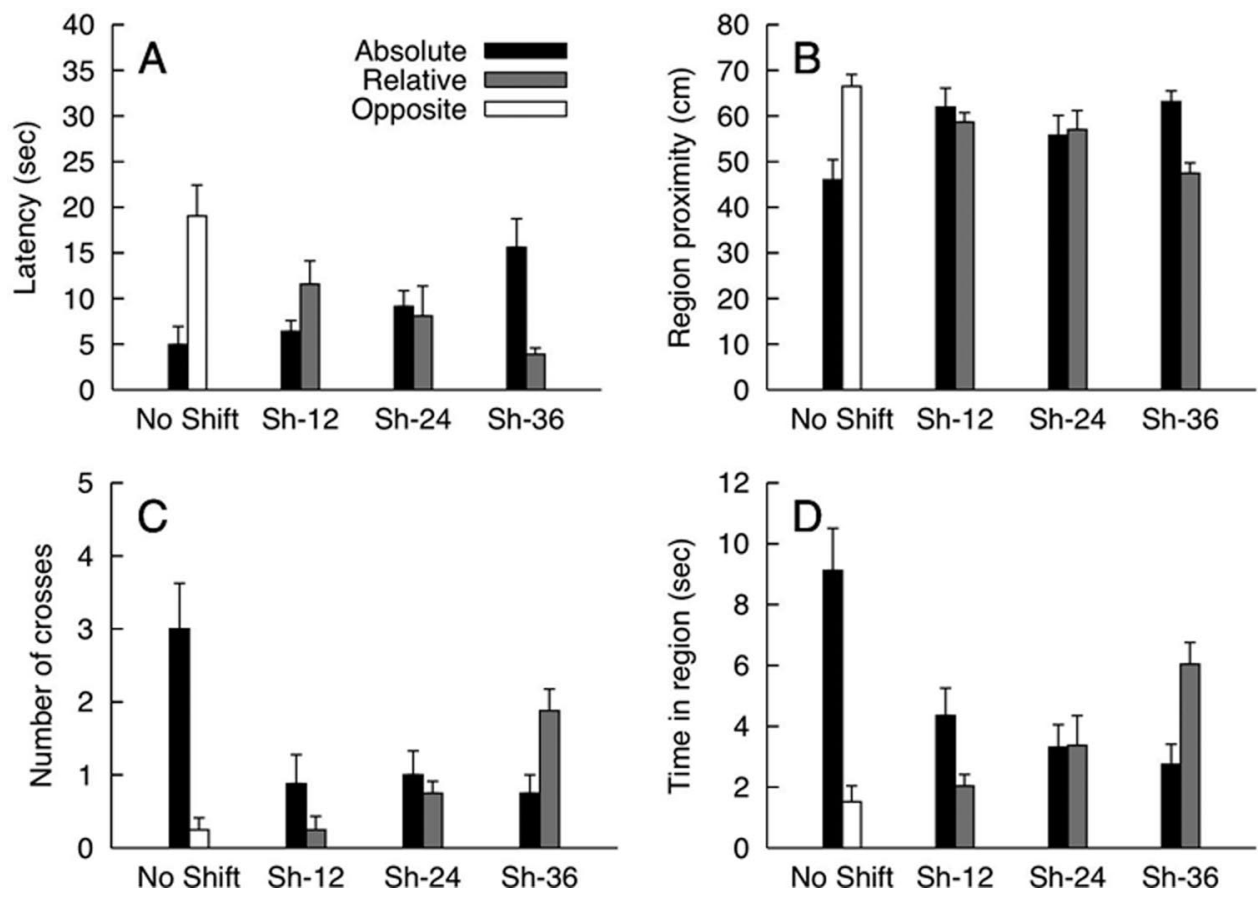

Figure 2. Probe trial dependent measures $(M+S E M)$ for each group. A: Latency to enter the $66 \mathrm{~cm}$ diameter circular region around the two locations of interest. B: Mean distance ("proximity") from the two critical locations. C: Number of times each critical location was crossed. D: Time spent in each of the two critical circular regions. 
differences among the mean dependent measures for each location largely support the conclusion that Shift-12 rats preferred the absolute over the relative location, only the effect for time in region (absolute $>$ relative) approached significance, $F(1,7)=5.01, p=$ .06 , all other $p s>.14$. A separate analysis using only data from the 7 rats that displayed a preference for the absolute location yielded a significant location effect in the expected direction for number of crosses (absolute $>$ relative), $F(1,6)=6.35$. The effect for time in region approached significance, $F(1,6)=5.28, p=.06$, however, the effects for the latency and proximity measures still failed to reach significance, $p$ s were .08 and .78 , respectively. Due to the importance of establishing the basic preferences for Shift-12 rats, Table 1 presents the means for each dependent measure broken down based on preference for the absolute versus relative location.

As a group, Shift-24 rats showed no clear preference for either the absolute or relative location (see Figure 2) and there were no significant location effects for any of the dependent measures, all $p s>.59$. Inspection of individual probe trial swim paths, however, indicates that individual rats displayed a clear location preference. Four rats displayed a preference for the absolute location and the other 4 rats displayed a preference for the relative location (representative swim paths are shown in the Supplementary Figure). Location means for each dependent measure broken down by location preference are shown in Table 1. Although the rather small number of rats displaying each preference makes it difficult to detect statistically significant location effects within groupings based on preference, we note that rats showing a preference for the absolute location navigated significantly closer to the absolute location, $F(1,3)=15.71$ and rats showing a preference for the relative location entered the relative region significantly faster than the absolute region, $F(1,3)=27.82$. None of the other location effects were significant for either grouping, all $p \mathrm{~s}>.12$. Nonetheless, it is important to note that the numerical differences between measures for the two critical locations within each grouping are quite similar to the numerical differences for clear location preferences we have observed in our previous experiments.

Shift-36 rats displayed a clear preference for the relative location over the absolute location (see Figure 2) as was apparent in the swim paths of all rats in this group (a representative swim path is shown in the Supplementary Figure). Consistent with this evaluation, significant location effects were observed for all dependent measures; Shift-36 rats entered the relative region faster than the absolute region, $F(1,7)=11.87$, spent more time in the relative region, $F(1,7)=6.34$, crossed the relative location more fre- quently, $F(1,7)=6.52$, and navigated closer to the relative location, $F(1,7)=13.59$.

The following analyses were conducted to address whether the location effects (absolute vs. relative/opposite) differed for the No Shift and shift groups. It was not possible to conduct an analysis with number of training trials and group (shift vs. No Shift) as factors because these factors were not completely crossed. Therefore, we conducted three separate two-way ANOVAs with location as a within-subjects factor. Each analysis included the No Shift group and one of the shift groups with group representing a single between-subjects factor for each analysis. For the No Shift versus Shift-12 analyses there were significant interactions for time in region, location crosses, and average proximity, all $p \mathrm{~s}<.03$. The interaction for latency to enter the critical regions was not significant, $F(1,14)=2.44, p=.14$. For the No Shift versus Shift-24 analyses there were significant interactions for latency, time in region, and location crosses, all $p \mathrm{~s}<.03$. The interaction for average proximity did not reach significance, $F(1,14)=3.51, p=$ .08. For the No Shift versus Shift-36 analyses there were significant interactions for all four dependent measures, all $p \mathrm{~s}<.001$. These significant interactions indicate that the location effects for each of the shift groups differed from those of the No Shift group. The series of simple location effects described above suggest that, as a whole, the two-way interactions noted between the No Shift group and the various shift groups occurred despite different patterns of location effects within the shift groups. For the Shift-12 group, the interactions can largely be attributed to differences between the Shift-12 and No Shift groups in terms of magnitude, but not direction, of the location effects. For the Shift- 24 group the interactions can be attributed to the presence of significant location effects in No Shift, whereas there were no compelling numerical or statistically significant location effects for the Shift-24 group as a whole. For the Shift-36 group the interactions are attributable to differences in the direction of significant location effects for the No Shift and Shift-36 group, with the No Shift group displaying a preference for the absolute location and the Shift-36 group displaying a clear preference for the relative location. The pattern of location effects for the shift groups clearly suggests that the location effect changed as a function of the number of training trials, and this change would appear to be related to the number of rats in each group that displayed a particular preference. To evaluate this impression we conducted separate ANOVAs limited to the shift groups, with number of training trials $(12,24$, or 36$)$ as a between-subjects factor and location (absolute vs. relative) as a

Table 1

Means for Each Dependent Measure During the No Platform Probe Trial for the Shift-12 and Shift-24 Groups

\begin{tabular}{|c|c|c|c|c|c|c|c|c|c|c|}
\hline \multirow[b]{2}{*}{ Group } & \multirow[b]{2}{*}{ Preference } & \multirow[b]{2}{*}{$n$} & \multicolumn{2}{|c|}{ Latency (s) } & \multicolumn{2}{|c|}{ Time (s) } & \multicolumn{2}{|c|}{ Crosses } & \multicolumn{2}{|c|}{ Proximity (cm) } \\
\hline & & & Absolute & Relative & Absolute & Relative & Absolute & Relative & Absolute & Relative \\
\hline Shift-12 & Place & 7 & 6.00 & 12.81 & 4.64 & 2.02 & 1.00 & 0.14 & 61.48 & 59.61 \\
\hline Shift-12 & Direction & 1 & 9.17 & 3.00 & 2.33 & 2.17 & 0.00 & 1.00 & 65.38 & 51.99 \\
\hline Shift-24 & Place & 4 & 5.33 & 10.83 & 4.62 & 2.54 & 1.50 & 0.50 & 49.59 & 64.96 \\
\hline Shift-24 & Direction & 4 & 12.92 & 5.33 & 2.00 & 4.21 & 0.50 & 1.00 & 61.99 & 49.07 \\
\hline
\end{tabular}

Note. Means are separated on the basis of whether rats showed a preference for true place navigation (to the absolute location) or directional responding (to the relative location). Values for the No Shift and Shift-36 rats are not presented because all rats in these groups showed either a preference place navigation (No Shift rats) or directional responding (Shift-36 rats), thus, the mean values for these groups are already represented in Figure 2. 
within-subjects factor. There were significant Location $\times$ Trials interactions for latency to enter the regions of interest, time in each region, and number of times each location was crossed, all $p \mathrm{~s}<$ .02 . The interaction for proximity to each location was not significant, $F(2,21)=2.01, p=.14$. Given the individual location effects described above, this interaction can perhaps be best characterized as resulting from an initial preference for the absolute location by a majority of rats which yielded to a preference for the relative location with additional training.

\section{Discussion}

The results obtained here provide evidence that true place navigation is observed relatively early in training, but yields to a preference for directional responding with additional training in a variant of the Morris water task in which the pool is not a prominent cue. When the pool was relocated for the probe trial most rats given 12 training trials navigated to and searched at the precise spatial location of the platform in the room reference frame, whereas all rats given 36 training trials preferred the relative spatial location of the platform within the pool. As a group, rats given an intermediate number of training trials (24) showed no clear preference for either the relative or the absolute spatial location of the platform, however, individual rats showed clear a preference for one location over the other. These data illustrate a systematic shift in preference from place navigation to directional responding as a function of training.

In contrast to the standard water task in which the pool is a prominent feature of the environment, the pool utilized in this study was filled nearly to the top with water and was surrounded by clear Plexiglas to prevent escape from the pool. Our previous observations in the standard water task (Akers et al., 2007; Hamilton et al., 2007, 2008) collectively demonstrated that directional responding predominates regardless of the amount of training. The full-pool variant of the task used here represents the only situation identified thus far in which a preference for place navigation is observed (Hamilton et al., 2008). The present results indicate that this preference is transient and begins to yield to a preference for directional responding within 12 to 24 additional training trials. These observations are of some importance, particularly because we previously reported that animals given 12 to 24 training trials display a preference for place navigation over directional responding in this variant of the water task. In our previous report (Hamilton et al., 2008, Experiment 6), rats were tested both after 12 and after 24 trials (i.e., the number of trials was a within-subjects factor) and a significant preference for place navigation was observed. Because no significant differences were observed across tests the data were collapsed for analysis. On collection of the present data we reexamined our previous results and noted that the place preference was more robust, numerically, after 12 trials than after 24 trials, and the preference was not significant for the test conducted after 24 trials. Thus, our prior results taken with the present findings indicate that a preference for place navigation diminishes or disappears with continued training. This observation holds some practical importance in that future studies using the full-pool variant of the water task reported by Hamilton et al. (2008) should be designed and evaluated with the transience of the place navigation preference in mind. The optimal conditions for a preference for place navigation are apparently only present early in training; however, it may be possible to achieve more robust place navigation using other procedures. Given the apparent shift from place to direction as a function of training it seems reasonable to expect that giving fewer than 12 training trials might enhance the place navigation preference reported here. The number of training trials used here was selected because rats begin to take direct trajectories to the platform and reach asymptotic levels of performance in the full-pool variant of the task in about 10 to 12 trials. Conducting fewer blocks of training is not advisable because 8 trials is not sufficient to achieve direct navigation to the platform, however, future studies could improve, although perhaps only slightly, by adopting a criterion for advancement to the test trial such that it is conducted as soon as possible after direct trajectories emerge. With this in mind, we evaluated the training swim paths during the final training trial block for the Shift-12 group and noted that 6 of the rats first navigated directly to the platform during the penultimate or final trial and the remaining 2 took direct paths on each of the last 3 trials. Thus, the present results for the Shift-12 group probably approximate what would be observed if a minimum criterion of 1 to 2 direct trajectories to the platform were adopted. In our opinion, obtaining more robust place navigation is more likely to be achieved through identification of the basic factors related to stimulus control that differentiate place and directional responding.

A better understanding of place and directional navigation in the water task, and the shift from one to the other, will benefit from studies using cue-controlled environments (Devan et al., 2002; Prados \& Trobalon, 1998; Rodrigo, Chamizo, McLaren, \& Mackintosh, 1997) to address questions regarding how distal stimuli control these forms of navigation as well as how one comes to predominate another. Of course, the preference for place navigation observed in the full-pool variant of the water task may be related to the fact that more of the distal cue environment is visible compared to when the pool is a prominent cue (Hamilton et al., 2008). The distal environment is also visible from a greater proportion of the pool because the pool wall does not completely obscure portions of the distal environment when the animal is in close proximity to the pool boundary. It is of some importance to emphasize that the increases in the available distal cue environment in the full-pool task is not accompanied by enhanced learning, but rather by a decrease in the rate of learning compared to the standard variant of the task as demonstrated by Hamilton et al. (2008) and supported by the findings of the present experiment. These observations also suggest that the pool wall is an important determinant of behavior in the water task even though it provides no cues that disambiguate the location of the platform and obscures other cues to do disambiguate spatial location (Hamilton et al., 2007, 2008). One possibility that could potentially be important for increasing our understanding of place and directional navigation is that navigation in the standard water task may involve a process whereby locations within the pool reference frame are disambiguated by the distal visual cues (Hamilton et al., 2008). Viewed in this way, directional navigation when the pool is repositioned occurs because animals navigate to the location within the pool reference frame that is the most consistent with the location, as defined by the distal cue reference frame, reinforced during training. Eliminating the pool almost entirely creates a situation in which navigation is based primarily on the distal cue reference frame, and as a result, navigation to precise locations in the distal 
cue reference frame is supported. Although the pool wall was reduced in the present experiment, a small portion of it remained visible and the Plexiglas barrier could have also been detected. Although the pool cues themselves were not changed during training, it is possible that they gradually acquired control as a function of training such that early during training navigation was primarily controlled by the distal cues, whereas later in training navigation was controlled by both distal cues and apparatus cues. Although such a possibility may seem a trivial explanation of the present data, it is important to recognize its importance because tasks used for measurement of navigation typically have detectable apparatus cues that do not disambiguate spatial locations but nonetheless provide an important proximal frame of reference. Thus, the possibility that shifts in strategy may reflect shifts in how apparatus cues control navigation and interact with distal cues will be important to consider in future studies. Unfortunately, directly testing the idea that the shift in control by the distal cues observed here reflects increasing control by the pool cues, rather than a pure change in control by distal cues, will be difficult because completely eliminating the cues associated with the pool is not possible. ${ }^{1}$ One approach currently being explored in our laboratory involves testing humans in a computerized (virtual) version of the Morris water task (e.g., Hamilton, Driscoll, \& Sutherland, 2002; Hamilton \& Sutherland, 1999; Sutherland \& Hamilton, 2004) in which the pool cues can be completely eliminated while still constraining movement within the perimeter of the pool.

The shift in preference reported here bears some similarity to data showing that rats shift from performance of place navigation to performance of simple motor responses with continued training in dry land mazes (Chang \& Gold, 2003; Hicks, 1964; Packard \& McGaugh, 1996; Ritchie, Aeschliman, \& Pierce, 1950), although we acknowledge that there may be no substantive parallel between the present data and those obtained in dry mazes. For example, simple motor responses are not a major constituent process involved in the Morris water task and the shift from place to response in dry mazes differs in that it likely involves a complete change in the type of controlling stimuli. For example, Ritchie et al. argued that early control by exteroceptive stimuli yields to increasing control by interoceptive stimuli with continued training. In contrast, both place and directional responding in the water task are controlled by distal visual stimuli, thus, the shift from place to directional responding reflects a change in how the relevant distal room and apparatus stimuli control navigation. Further, Skinner et al. (2003) suggested that prior studies claiming a shift from place navigation to simple motor responding in dry land mazes may actually reflect a shift from directional responding to motor responding because directional responding was not clearly ruled out. The main point we wish to emphasize regarding the similarity between our water task data and extant dry maze data is that both indicate substitution of apparent place navigation with another form of responding. Given that directional responding has not been ruled out in earlier reports using dry mazes, however, the present results may be the first to unambiguously demonstrate a shift from true place navigation to another form of responding.

In summary, the present results indicate that any preferences for place navigation are transient and weak in comparison to directional responding, which fits nicely with previous data from our laboratory using the Morris water task (Akers et al., 2007; Hamilton et al., 2007, 2008; Weisend et al., 1995) and data from dry land maze studies (Blodgett et al., 1949; Horne, Martin, Harley, \& Skinner, 2007; Skinner et al., 2003; Stringer, Martin, \& Skinner, 2005). Additional work using similar manipulations to those reported here will be important in further elucidating the processes involved in place navigation and directional responding, and how preferences for these forms of navigation are established and altered during training.

\footnotetext{
${ }^{1}$ Completely filling the tank and removing the clear Plexiglas enclosure would simply provide a ready means of escape from the pool, thus, some barrier around the perimeter of the pool is necessary.
}

\section{References}

Akers, K. G., Candelaria, F. T., \& Hamilton, D. A. (2007). Preweanling rats solve the Morris water task via directional navigation. Behavioral Neuroscience, 121, 1426-1430.

Blodgett, H. C., McCutchan, K., \& Mathews, R. (1949). Spatial learning in the T-maze: The influence of direction, turn, and food location. Journal of Experimental Psychology, 39, 800-809.

Chamizo, V. D., Aznar-Casanova, J. A., \& Artigas, A. A. (2003). Human overshadowing in a virtual pool: Simple guidance is a good competitor against locale learning. Learning and Motivation, 34, 262-281.

Chamizo, V. D., Sterio, D., \& Mackintosh, N. J. (1985). Blocking and overshadowing between intra-maze and extra-maze cues: A test of the independence of locale and guidance learning. Quarterly Journal of Experimental Psychology B, 37, 235-253.

Chang, Q., \& Gold, P. E. (2003). Intra-hippocampal lidocaine injections impair acquisition of a place task and facilitate acquisition of a response task in rats. Behavioural Brain Research, 144, 19-24.

Devan, B. D., Petri, H. L., Mishkin, M., Stouffer, E. M., Bowker, J. L., Yin, P. B., et al. (2002). A room with a view and a polarizing cue: Individual differences in the stimulus control of place navigation and passive latent learning in the water maze. Neurobiology of Learning and Memory, 78, 79-99.

Gallagher, M., Burwell, R., \& Burchinal, M. (1993). Severity of spatiallearning impairment in aging-development of a learning index for performance in the Morris water maze. Behavioral Neuroscience, 107, 618-626.

Hamilton, D. A., Akers, K. G., Johnson, T. E., Rice, J. P., Candelaria, F. T., Sutherland, R. J., et al. (2008). The relative influence of place and direction in the Morris water task. Journal of Experimental Psychology: Animal Behavior Processes, 34, 31-53.

Hamilton, D. A., Akers, K. G., Weisend, M. P., \& Sutherland, R. J. (2007). How do room and apparatus cues control navigation in the Morris water task?: Evidence for distinct contributions to a movement vector. Journal of Experimental Psychology: Animal Behavior Processes, 33, 100-114.

Hamilton, D. A., Driscoll, I., \& Sutherland, R. J. (2002). Human place learning in a virtual Morris water task: Some important constraints on the flexibility of place navigation. Behavioural Brain Research, 129, $159-170$.

Hamilton, D. A., Rosenfelt, C. S., \& Whishaw, I. Q. (2004). Sequential control of navigation by locale and taxon cues in the Morris water task. Behavioural Brain Research, 154, 385-397.

Hamilton, D. A., \& Sutherland, R. J. (1999). Blocking in human place learning: Evidence from virtual navigation. Psychobiology, 27, 453461.

Hicks, L. H. (1964). Effects of overtraining on acquisition and reversal of place and response learning. Psychological Reports, 15, 459-462.

Horne, M. R., Martin, G. M., Harley, C. W., \& Skinner, D. M. (2007). Where am I? Distal cue use requires sensitivity to start location change in the rat. Journal of Experimental Psychology: Animal Behavior Processes, 33, 92-99. 
Morris, R. G. M. (1981). Spatial localization does not require the presence of local cues. Learning and Motivation, 12, 239-260.

O'Keefe, J., \& Nadel, L. (1978). The hippocampus as a cognitive map. Oxford, England: Clarendon.

Packard, M. G., \& McGaugh, J. L. (1996). Inactivation of hippocampus or caudate nucleus with lidocaine differentially affects expression of place and response learning. Neurobiology of Learning and Memory, 65, 65-72.

Prados, J., \& Trobalon, J. B. (1998). Locating an invisible goal in a water maze requires at least two landmarks. Psychobiology, 26, 42-48.

Redhead, E. S., Roberts, A., Good, M., \& Pearce, J. M. (1997). Interaction between piloting and beacon homing by rats in a swimming pool. Journal of Experimental Psychology: Animal Behavior Processes, 23, $340-350$.

Restle, F. (1957). Discrimination of cues in mazes: A resolution of the "place-vs.-response" question. Psychological Review, 64, 217-228.

Ritchie, B. F., Aeschliman, B., \& Pierce, P. (1950). Studies in spatial learning. VIII. Place performance and the acquisition of place dispositions. Journal of Comparative and Physiological Psychology, 43, 7385.

Roberts, A. D. L., \& Pearce, J. M. (1999). Blocking in the Morris swimming pool. Journal of Experimental Psychology: Animal Behavior Processes, 25, 225-235.

Rodrigo, T., Chamizo, V. D., McLaren, I. P. L., \& Mackintosh, N. J. (1997). Blocking in the spatial domain. Journal of Experimental Psychology: Animal Behavior Processes, 23, 110-118.
Skinner, D. M., Etchegary, C. M., Ekert-Maret, E. C., Baker, C. J., Harley, C. W., Evans, J. H., et al. (2003). An analysis of response, direction, and place learning in an open field and $\mathrm{T}$ maze. Journal of Experimental Psychology: Animal Behavior Processes, 29, 3-13.

Stringer, K. G., Martin, G. M., \& Skinner, D. A. (2005). The effects of hippocampal lesions on response, direction, and place learning in rats. Behavioral Neuroscience, 119, 946-952.

Sutherland, R. J., \& Hamilton, D. A. (2004). Rodent spatial navigation: At the crossroads of cognition and movement. Neuroscience Biobehavioral Reviews, 28, 687-697.

Tolman, E. C. (1948). Cognitive maps in rats and men. Psychological Review, 55, 189-208.

Tolman, E. C., Ritchie, B. F., \& Kalish, D. (1946). Studies in spatial learning. II. Place learning versus response learning. Journal of Experimental Psychology, 3, 221-229.

Watson, J. B. (1907). Kinesthetic and organic sensations: Their role in the reaction of the white rat to the maze. Psychological Monographs, 8 , $1-101$.

Weisend, M. P., Klein, R. L., Hoesing, J. M., Astur, R. S., Koerner, A., McDonald, R. J., et al. (1995). Morris water task: Which cues define locations? Society for Neuroscience Abstracts, 21, 1939.

Received April 5, 2008 Revision received July 3, 2008 Accepted July 4, 2008

\section{Correction to Darredeau et al. (2009)}

In the article "Competition Between Multiple Causes of a Single Outcome in Causal Reasoning," by Christine Darredeau, Irina Baetu, Andrew G. Baker, and Robin A. Murphy (Journal of Experimental Psychology: Animal Behavior Processes, 2009, Vol. 35, No. 1, pp. 1-14), the URL provided for the supplemental material was incomplete. The complete URL is http://dx.doi.org/10.1037/a0012699.supp

DOI: $10.1037 / \mathrm{a} 0015627$ 